\title{
The Standard Model of Particle Physics
}

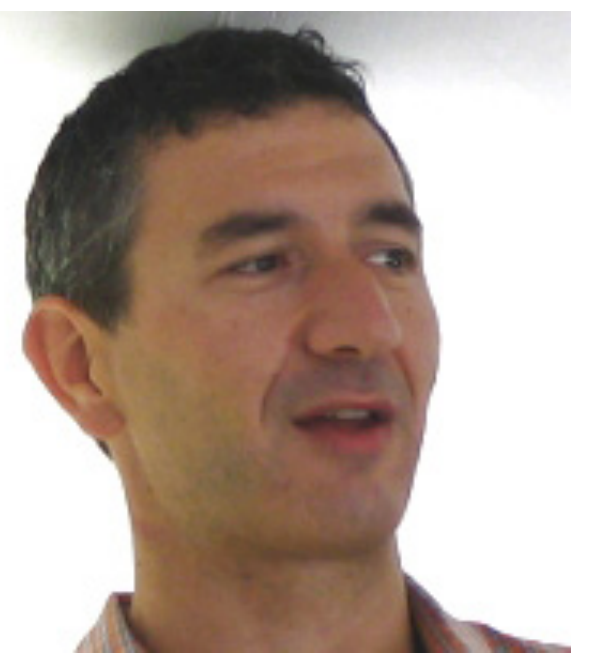

Andrea Romanino - Associate Professor, SISSA. The scientific interests include Standard Model and beyond, including supersymmetry, extra dimensions and grand unification; flavour and neutrino physics.

\begin{abstract}
These lectures provide a basic introduction to the Standard Model (SM) of particle physics. While there are several reasons to believe that the Standard Model is just the low energy limit of a more fundamental theory, the SM has been successfully tested at an impressive level of accuracy and provides at present our best fundamental understanding of the phenomenology of particle physics. The perspective I will take will not be historical, I will instead take advantage of our present understanding to find the most direct logical motivations.
\end{abstract}




\title{
The Standard Model of Particle Physics
}

\author{
Andrea Romanino
}

SISSA/ISAS and INFN, I-34151 Trieste, Italy

\section{Introduction}

These lectures provide a basic introduction to the Standard Model (SM) of particle physics. While there are several reasons to believe that the Standard Model is just the low energy limit of a more fundamental theory, the SM has been successfully tested at an impressive level of accuracy and provides at present our best fundamental understanding of the phenomenology of particle physics. The perspective I will take will not be historical, I will instead take advantage of our present understanding to find the most direct logical motivations. As the level of the audience is quite diverse, I will summarize (in a concise, qualitative, and pragmatic way) the main theoretical preliminaries needed to make sense of what will follow. The hope is that a part of these lectures could be useful even to undergraduate students, that people who are already familiar with QFT and gauge theories can also benefit from them, and people in between can have a first impact with the tools involved in a non qualitative treatment. I will systematically use natural units, in which $c=\hbar=1$.

Let us start with the very basic facts.

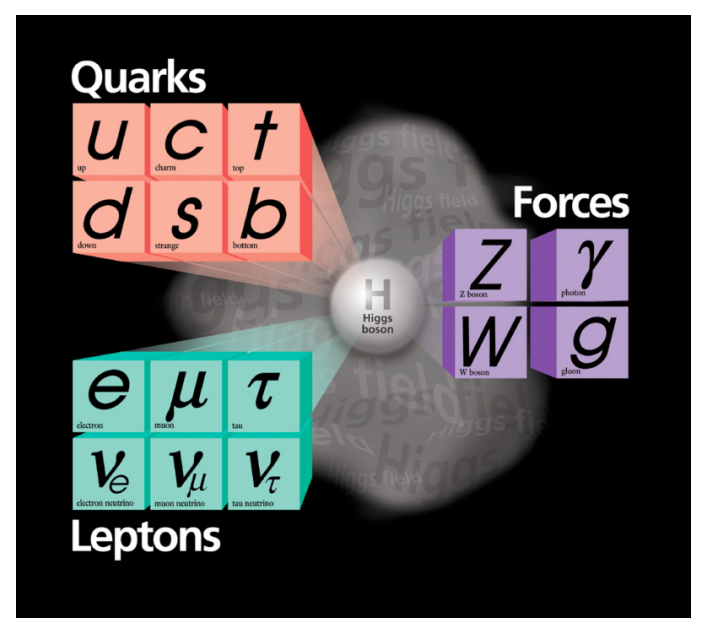

Figure 1: The SM particle content The main ingredients of the SM are shown in Fig. 1. The particles involved are characterized by their spin, their mass, and the quantum numbers (charges) determining their interactions. The fermion content $($ spin $=1 / 2)$ is organized in three families with identical quantum numbers and different masses. The heavier families are unstable and decay into the lightest one, which makes up most of the ordinary matter (us). The four fermions in each family are distinguished by their charges under strong and electromagnetic interactions. Two of them are quarks, which are charged under the strong interactions, and two are leptons, which are not. The two quarks have electromagnetic charges $2 / 3$ ("up" quarks) and $-1 / 3$ ("down" quarks) respectively, and the two leptons have charges -1 (charged, or "down" leptons) and 0 (neutrinos, or "up" leptons), in units in which the electron 
charge is -1 . The neutrinos are peculiar from two, probably theoretically related, points of view: they are neutral under both the strong and the electromagnetic interactions (they feel weak interactions, though) and they are at least six order of magnitudes lighter than all the other SM fermions. The masses of the SM fermions span a range going from the sub-eV neutrino masses to the $1.7 \cdot 10^{2} \mathrm{GeV}$ top mass [1]. They exhibit quite a peculiar structure, with the masses of the different families being hierarchically separated. Each fermion is associated to two so-called chiralities. Chirality is conserved for massless fermions, in which case the chirality coincides with the helicity. That is why the two possible chiralities are called left-handed and righthanded. From a theoretical point of view, chirality, by definition, distinguishes the two irreducible representations of the Lorentz group that can be used to describe spin $1 / 2$ fermions. Massive charged fermions are necessarily described by two components of different chiralities combined in what is called a Dirac spinor. As for neutrinos, only the left-handed chirality has been observed so far. This can be elegantly understood in terms of the quantum numbers of a possible right-handed component, but the argument goes beyond the scope of these lectures.

The SM interactions are associated to the exchange of four vector bosons (spin = 1). The photon mediates electromagnetic interactions, the gluon strong interactions, the $Z$ and $W$ weak interactions. The photon and the gluons are massless, while the $Z$ and the $W$ are massive, which is the reason why weak interactions are weak at low energy (they are suppressed by powers of $E / M_{Z, W}$, where $E$ is the energy of the process). Despite their weakness, they give rise to distinctive signatures because they violate parity $P$, charge conjugation $C$, their combination $C P$, time-reversal $T$, and family number, which all are symmetries of the electromagnetic and strong ${ }^{1}$ interactions. In particular, the decay of heavier into lighter families is due to weak interactions.

The description outlined above holds at relatively low energy and has long been known before the SM was invented. The SM description becomes necessary when processes involving higher energies are considered. The transition from the low energy "effective" regime and the SM regime takes place around the electroweak scale $v \approx 174 \mathrm{GeV}$. Above this scale, nature exhibits a higher degree of symmetry. The electromagnetic and weak interactions become indistinguishable and are unified in the "electroweak" interaction. The left-handed chirality components of up and down fermions also become indistinguishable and are unified in electroweak doublets. The electroweak scale is where such an "electroweak" symmetry breaks. Together with the QCD and the Planck scale, it is one of the fundamental scales of nature known at present. The mechanism through which the electroweak symmetry breaks is well established, also experimentally, and is called spontaneous symmetry breaking. It is through such a mechanism that the fermions and the massive gauge bosons acquire

\footnotetext{
${ }^{1}$ In the limit in which non-perturbative effects associated to the vacuum structure of QCD are neglected.
} 
a mass proportional to the electroweak scale ${ }^{2}$. It is still not know, however, what is the mechanism triggering the spontaneous breaking. The SM encodes the simplest (both from the theoretical and phenomenological consistency point of view) option: the Higgs mechanism [2]. Such a mechanism postulates the existence of a spin $=0$ field, the Higgs field. Unlike all other fields, that require energy to be switched on, the Higgs field is "on" even in the ground state, where it permeates space-time. It is through their interactions with the Higgs field that the SM massive particles acquire their masses, proportional to the coupling to the Higgs. The identification of the mechanism responsible for the electroweak symmetry breaking and the stability of the weak scale compared to the Planck scale are two central issues in today's particle physics and are two of the most important missions of the LHC.

In order to go beyond the qualitative picture presented so far, I need to introduce some important theoretical tools, at least at a very basic level. The key tools are gauge theories and their spontaneous breaking. In Section 2 I will introduce gauge symmetries, which allow to define the SM gauge sector in Section 3. Spontaneous breaking will be introduced in Section 4, which allows to introduce the SM Higgs and Yukawa sectors in Section 5. In Section 6 we will discuss the phenomenological implications of the SM and mention the open problems.

\section{Gauge (and global) transformations}

The SM is first of all a quantum field theory (QFT). In QFT, particles are associated to fields $\phi_{i}(x), i=1 \ldots n$ depending on the space-time coordinates $x=\left(x^{0}, x^{1}, x^{2}, x^{3}\right)$. We consider only fields with spin $s=0,1 / 2,1$ (no gravity), the only ones needed for the SM, and the only ones for which we know how to write a theoretically consistent QFT. Their dynamics is determined by an action $S$ written in terms of a Lagrangian density $\mathcal{L}(x)$ (which I will simply call "Lagrangian") with dimension 4 in energy. The SM Lagrangian is Lorentz-invariant and local, and it can be written as a sum of monomials in the values of the fields and their derivatives (up to two) in a given space-time point:

$$
S=\int d^{4} x \mathcal{L}(x), \quad \mathcal{L}(x)=\sum_{k} c_{k} \mathcal{O}_{k}(x) .
$$

In particular, the Lagrangian will contain: a term constant in the fields, which is not physical as long as gravity is not taken into account; terms linear in the fields, which can be reabsorbed by a shift redefinition of the fields $\phi(x) \rightarrow \phi(x)+c$; terms bilinear in the fields, the "free" Lagrangian $\mathcal{L}_{\text {free }}$, which account for the free propagation of the fields and define their dimensions in energy ( 1 for the bosons and $3 / 2$ for the fermions); terms with at least three fields, the "interaction" Lagrangian $\mathcal{L}_{\text {int }}$, which

\footnotetext{
${ }^{2}$ Except possibly the neutrinos, whose masses are likely to be quadratic in the electroweak scale.
} 
account for field interactions. In a perturbative regime, the amplitude of any physical process can be expressed as an expansion in $\mathcal{L}_{\text {int }}$ represented (at a given order) by a set of Feynman diagrams. The simplest example of interacting QFT involves a single real, scalar field $\varphi(x)$ with Lagrangian $\mathcal{L}=(\partial \phi)^{2} / 2-m^{2} \phi^{2} / 2-\lambda \phi^{4} / 4$.

The dimension of the coefficients $c_{k}$ in eq. (1) is important, as it determines the properties of the corresponding interaction. Terms with negative dimension, $c=1 / \Lambda^{D}, D>0$, where $\Lambda$ is an energy scale, decouple at sufficiently low energy $E$, where their role is suppressed by $(E / \Lambda)^{D}$. They are called "non-renormalizable". Only "renormalizable" terms with non-negative dimension of the coefficients are therefore relevant at low enough energy. The presence of non-renormalizable terms becomes important at high energy, where they end up making the theory incalculable. As the amplitudes grow with $(E / \Lambda)^{D}$, in fact, higher order in perturbative expansions will eventually become as important as the lower orders: the theory becomes non perturbative. This is signaled by the fact that the unitarity of the theory appears to be violated at the perturbative level. Therefore, non-renormalizable terms can be tolerated only in the context of an effective theory valid up to a certain energy scale ("cutoff") $\Lambda$. At the scale $\Lambda$, such terms should be accounted for by renormalizable interactions, if the theory is to remain perturbative and calculable. Such theoretical considerations played an important role in the development of the SM. The latter, as we will see, was born from the need to express the non-renormalizable four-fermion Fermi interaction accounting for weak interactions at low energy in terms of a renormalizable theory.

Let us now come to the role of symmetries. We will be dealing with $\mathrm{SU}(N)$ and $\mathrm{U}(1)$ symmetry groups only. The generators $t$ of the group parametrize infinitesimal transformations $u \approx \mathbf{1}-i \epsilon t$, form a Lee algebra, $\left[t_{1}, t_{2}\right]=i t_{3},\left[t_{1},\left[t_{2}, t_{3}\right]\right]+\left[t_{2},\left[t_{3}, t_{1}\right]\right]+$ $\left[t_{3},\left[t_{1}, t_{2}\right]\right]=0$, and in the case of $\mathrm{SU}(N)$ are Hermitian traceless matrices. A standard choice of $\mathrm{SU}(N)$ generators is $t_{a}=\sigma_{a} / 2, a=1,2,3$, for the case $N=2$, where $\sigma_{a}$ are the Pauli matrices; $t_{A}=\lambda_{A} / 2, A=1 \ldots 8$, for the case $N=3$, where $\lambda_{A}$ are the Gell-Mann matrices; $t_{i}, i=i \ldots N^{2}-1$, with $\operatorname{tr}\left(t_{i} t_{j}\right)=\delta_{i j} / 2$, in the general case. The structure constants $f_{i j k}$ are defined by $\left[t_{i}, t_{j}\right]=i f_{i j k} t_{k}$ and are antisymmetric.

In quantum mechanics a continuous symmetry group is represented by unitary transformations on the states of the system that commute with the Hamiltonian. The generators correspond to conserved quantities. In QFT, we consider symmetries whose action on the states corresponds to a transformation of the fields $\phi_{i}(x) \rightarrow$ $U_{i j} \phi_{j}(x)$ that is a symmetry of the Lagrangian. If $U$ does not depend on the space-time point $x$, we call the symmetry "global", or "rigid". The generators of the symmetry, or, more precisely, their actions $T$ on the quantum states, are given by Noether's theorem in terms of conserved currents,

$$
T=\int d \boldsymbol{x} j^{0}, \quad j^{\mu}=\frac{\partial \mathcal{L}}{\partial\left(\partial_{\mu} \phi\right)} \delta \phi, \quad \partial_{\mu} j^{\mu}=0
$$


where $\delta \phi$ is the infinitesimal change of $\phi$ associated to the generator $t$. A global symmetry is a true symmetry, meaning that it relates physically inequivalent states (multiplets) with same mass and spin and their couplings. The classic example of global symmetry in QFT is isospin, which acts on the doublet $Q=(u, d)^{T}$ made of the up and down quark fields. In the limit $m_{u}=m_{d}=0$ and neglecting electromagnetic interactions, the $\mathrm{SU}(2)$ isospin transformation $Q \rightarrow U Q$ is a symmetry of the QCD Lagrangian for $u, d$. The isospin currents are $j_{a}^{\mu}=\bar{Q} \gamma^{\mu}\left(\sigma_{a} / 2\right) Q$ (one for each generator). The isospin symmetry relates masses and couplings of inequivalent states. The experimental proof that isospin is indeed an (approximate) symmetry comes from the fact that the light hadrons do organize themselves into isospin multiplets. For example, the proton and the neutron make up a isospin $1 / 2$ doublet with $m \approx 940 \mathrm{MeV}$, the pions an isospin 1 triplet with $m \approx 140 \mathrm{MeV}$. The effective pion-nucleon couplings are also related by the isospin symmetry.

Gauge transformations are not symmetries in the sense in which global symmetries are. Their action on the fields does depend on the space-time point and from the physical point of view they do not relate inequivalent physical states. They relate on the contrary equivalent field configurations, whose redundance should be taken into account and factored out.

In order to have a feeling of how gauge symmetries come about, let us consider the prototypical gauge theory, quantum electrodynamics (QED). The gauge symmetry is first of all a property of the classical theory. We know that the physical degrees of freedom of electrodynamics are the electromagnetic field $F_{\mu \nu}$ and the current $j^{\mu}$, in terms of which Maxwell equations are formulated. On the other hand, $F_{\mu \nu}$ is not a good dynamical variable, in particular it does not allow the Maxwell equations to be obtained from a variational principle. On the other hand, it is possible to express $F_{\mu \nu}$ in terms of the vector potential $A_{\mu}$ as $F_{\mu \nu}=\partial_{\mu} A_{\nu}-\partial_{\nu} A_{\mu}$. The equations of motion can then be derived from the Lagrangian $\mathcal{L}=-\frac{1}{4} F_{\mu \nu} F^{\mu \nu}-j^{\mu} A_{\mu}$. On the other hand, $A_{\mu}$ is not a physical variable, as two fields related by the gauge transformation $A(x)_{\mu} \rightarrow A_{\mu}(x)+\partial_{\mu} \alpha(x)$ correspond to the same physical observable $F_{\mu \nu}$. Therefore, the gauge transformation just defines the equivalence of configurations corresponding to the same physical observable, it signals a redundancy in the degrees of freedom we are using.

Let us now consider the quantum theory and consider the case in which the electromagnetic current is associated to a single Dirac fermion $\psi$ with charge $Q e$, $j^{\mu}=e Q \bar{\psi} \gamma^{\mu} \psi$, where $e$ is the absolute value of the charge of the electron. The Lagrangian will accordingly be given by

$$
\mathcal{L}_{\mathrm{QED}}=-\frac{1}{4} F_{\mu \nu} F^{\mu \nu}-j^{\mu} A_{\mu}+\bar{\psi}\left(i \gamma^{\mu} \partial_{\mu}-m\right) \psi,
$$

where the free Lagrangian of the Dirac spinor has also been added. We can now check explicitly that: 1) the electromagnetic current $j^{\mu}$ turns out to be the Noether current associated to the $\mathrm{U}(1)$ global symmetry under which $\psi(x) \rightarrow e^{-i e Q \alpha} \psi(x)$, which 
therefore can be thought to account for the conservation of the electric charge; and 2 ) the $U(1)$ symmetry turns out to be also related to gauge transformations. Let us remember in fact that we want the Lagrangian to be invariant with respect to gauge transformations of the vector field, or we would be able to distinguish equivalent configurations. It turns out that the Lagrangian is invariant provided that $\psi$ transforms according to the local version of the global U(1) transformation above:

$$
A(x)_{\mu} \rightarrow A_{\mu}(x)+\partial_{\mu} \alpha(x), \quad \psi(x) \rightarrow e^{-i e Q \alpha(x)} \psi(x) .
$$

Gauge invariance can therefore be considered as the principle underlying charge conservation. In order to verify the invariance of the Lagrangian, is is convenient to define the "covariant" derivative

$$
D_{\mu}=\partial_{\mu}+i e Q A_{\mu}, \quad \text { such that } D_{\mu}\left(e^{-i e Q \alpha(x)} \psi(x)\right)=e^{-i e Q \alpha(x)}\left(D_{\mu} \psi(x)\right) .
$$

The QED Lagrangian can then be written as $\mathcal{L}_{\mathrm{QED}}=-\frac{1}{4} F_{\mu \nu} F^{\mu \nu}+\bar{\psi}\left(i \gamma^{\mu} D_{\mu}-m\right) \psi$.

One can go one step further and argue that not only gauge invariance is the principle underlying charge conservation but that the QED Lagrangian itself follows from the principle of gauge invariance. The point is that the Lagrangian in eq. (3) can be obtained by forcing the spinor free Lagrangian $\bar{\psi}\left(i \gamma^{\mu} \partial_{\mu}-m\right) \psi$, invariant under global $\mathrm{U}(1)$ transformations $\psi(x) \rightarrow e^{-i e Q \alpha} \psi(x)$, to be invariant under local $\mathrm{U}(1)$ transformations $\psi(x) \rightarrow e^{-i e Q \alpha(x)} \psi(x)$. The argument goes as follows. In order to make the derivative term invariant, one is forced to introduce a vector field $A_{\mu}$ transforming as in eq. (4). A gauge invariant kinetic term for $A_{\mu}$ can then be built in terms of the gauge invariant quantity $F_{\mu \nu}$. Gauge invariance also explains why the photon is massless, as a mass term for $A_{\mu}$ would break gauge invariance.

There is also a second reason to believe that gauge invariance should be regarded as a fundamental principle, related to the fact that the quantization of the photon field is not straightforward. I will not enter the details of this argument, but will just mention that promoting a vector field to a gauge field is the only known consistent way to quantize it, and that its quantization requires adding a "gauge fixing" term, for example $-\left(\partial_{\mu} A^{\mu}\right)^{2} /(2 \xi)$, to the Lagrangian in order to get rid of the redundant degrees of freedom.

Given the impressive success of QED as a gauge theory (both in confronting the experiment and in addressing the quantization of vector bosons), it is natural to consider the generalization of the gauge principle to a generic (compact, Lie) group. Particularly interesting is the case of non-Abelian groups, which leads to at least two qualitative differences with the simple Abelian case of QED: the vector bosons acquire self-interactions and the coupling (which in QFT depends on the energy scale of the process in which it is measured) may get stronger at lower scales. Non-Abelian gauge theories are therefore a candidate to describe strong interactions. Indeed, it turns out that strong interactions can be described by a gauge theory based on a $\mathrm{SU}(3)$ gauge 
group acting on a "color" degree of freedom, quantum chromodynamics (QCD). QCD will be addressed in the lectures by Michelangelo Mangano.

Let us then consider a general gauge theory. Having motivated the construction in some detail in the simple Abelian case, I will now just provide the result of the generalized construction in the form of a recipe to construct a general renormalizable gauge theory of spin $0,1 / 2,1$ fields in four space-time dimensions (up to non-perturbative effects), without any further proof or motivation. The ingredients that need to be specified are the following. 1) A compact gauge group $G$ (which specifies the vector field content). 2) The scalar fields $\phi_{i}, i=1 \ldots n_{s}$ and fermion fields $\psi_{i}, i=1 \ldots n_{f}$ (collectively denoted in the following as $\left.\Phi\right)$. 3) The transformations (quantum numbers) of the latter under the action of the gauge group. 4) A renormalizable Lagrangian $\mathcal{L}_{0}(\Phi, \partial \Phi)$ for the scalar and fermion fields and their derivatives symmetric under the global (space-time independent) transformations of the fields specified above. Before seeing how the Lagrangian is obtained in terms of the above ingredients, let us elaborate on the specification of the fermion field content and quantum numbers. The point is that the fermions are usually described in terms of Dirac spinors $\psi_{i}$ that, as we said in the introduction, combine two independent components with left and right chirality respectively, $\psi_{i L}=\left(1-\gamma_{5}\right) \psi_{i} / 2$ and $\psi_{i R}=\left(1+\gamma_{5}\right) \psi_{i} / 2$ (in a given convention for the gamma matrices), each being an irreducible representation of the Lorentz group. Now, the only requirement on the action of the gauge group on fermions is that it commutes with the Lorentz group. As a consequence, its most general action is not bound to transform $\psi_{i L}$ and $\psi_{i R}$ in the same way: they could have different quantum numbers. This is not the case in QED and QCD, or in any parity conserving theory of charged particles, but turns out to be the case for the SM. A theory like the SM, where the left and right chirality components of fermions transform under inequivalent representations of the gauge group is called "chiral". A chiral theory violates parity. Actually, something even more general could happen. Since the left and right chirality representations of the Lorentz group are conjugated to each other, $\psi_{i R}^{*}$ is left handed, as $\psi_{i L}$. Therefore, the most general action of the gauge group on the fermions could mix the $\psi_{i L}$ and $\psi_{i R}^{*}$ fields. This is not the case in the SM, but does happen in grand-unified theories. In summary, the fermion field content should be specified in terms of the left-handed fermions, by specifying the quantum numbers of both $\psi_{i L}$ and $\psi_{i R}^{*}$ (or equivalently $\psi_{i L}$ and $\psi_{i R}$ ).

Let us now construct the Lagrangian associated to the above ingredients by the gauge principle. The recipe is the following. Choose a basis of generators $t_{a}$ for the group $G$ as above and associate a real vector field $A_{\mu}^{a}$ and a field strength $F_{\mu \nu}^{a}=$ $\partial_{\mu} A_{\nu}^{a}-\partial_{\nu} A_{\mu}^{a}-g f_{a b c} A_{\mu}^{b} A_{\nu}^{c}$ to it. Define the covariant derivative $D_{\mu}=\partial_{\mu}+i g A_{\mu}^{a} T_{a}$, where $T_{a}$ is the action of the generator $t$ on the fields $\Phi$ (then given a gauge transformation $\Phi(x) \rightarrow U(x) \Phi(x)$ we have $F_{\mu \nu}^{a} T_{a} \equiv \mathbf{F}_{\mu \nu} \rightarrow U \mathbf{F}_{\mu \nu} U^{-1}$ and $D_{\mu}(U(x) \Phi(x))=$ 
$\left.U(x)\left(D_{\mu} \Phi(x)\right)\right)$. The gauge Lagrangian is then

$$
\mathcal{L}=-\frac{1}{4} F_{\mu \nu}^{a} F_{a}^{\mu \nu}+\mathcal{L}_{0}(\Phi, D \Phi)-\frac{1}{2 \xi}\left(\partial_{\mu} A_{a}^{\mu}\right)^{2}+\text { ghosts }
$$

where the first term is the so-called Yang-Mills Lagrangian for the vector fields, the derivative has been promoted to a covariant derivative, the next to last term fixes the gauge and the last term involves auxiliary, unphysical anticommuting scalars (ghosts) important to preserve unitarity, whose discussion goes well beyond the purpose of these lectures (they will not play a role in what follows). As a simple exercise one can recover the QED Lagrangian in eq. (3) from the free Lagrangian of a Dirac fermion. The gauge interactions between matter and gauge fields come from the terms involving the covariant derivative, i.e. from the kinetic terms in the Lagrangian $\mathcal{L}_{0}$ before gauging. In the case of a set of fermions $\Psi=\left(\psi_{1} \ldots \psi_{n_{f}}\right)^{T}$ subject to an action $\Psi \rightarrow T_{a} \Psi$ of the generators, the kinetic term $\bar{\Psi} i \partial^{\mu} \gamma_{\mu} \Psi$ gives

$$
\bar{\Psi} i \partial_{\mu} \gamma^{\mu} \Psi \rightarrow \bar{\Psi} i D_{\mu} \gamma^{\mu} \Psi=\bar{\Psi} i \partial_{\mu} \gamma^{\mu} \Psi-g A_{\mu}^{a} \bar{\Psi} \gamma^{\mu} T_{a} \Psi
$$

The gauge bosons self-interactions are $g f_{a b c} \partial_{\mu} A_{\nu}^{a} A^{b \mu} A^{c \nu}-\frac{1}{4} g^{2} f_{a b c} f_{a b^{\prime} c^{\prime}} A^{b \mu} A^{c \nu} A_{\mu}^{b^{\prime}} A_{\nu}^{c^{\prime}}$. All gauge interactions are determined in terms of a universal gauge coupling $g$. In the case in which the gauge group is made of several factors (as in the case of the SM, as we will see), there is one independent gauge coupling for each irreducible factor.

\section{The SM gauge interactions}

We are now ready (at last) to start presenting the Standard Model Lagrangian. As the SM is a gauge theory, it suffices to specify the ingredients listed above to define it completely. It is however instructive to outline some of the logic that lead to the choice of such a model (and ingredients) to describe electroweak interactions at energies of the order of the electroweak scale or higher. The way the SM emerges from the experimental information that was available when it was discovered can be considered (in quite a reductive way) as one of the nicest, and definitely most useful, model-building exercises ever [3]. Let us then review it, following a shortcut making use of some of our present knowledge.

Let us consider to begin with the fermion content of a single family of fermions, whose electromagnetic, strong, and weak interactions we want to describe in the context of a theoretically consistent theory that can be extrapolated at and beyond what we now know to be the electroweak scale. We then have the fermion fields $\nu, e, u$, d. QED and QCD can be accounted for by a gauge Lagrangian based on gauge groups $\mathrm{U}(1)_{\mathrm{em}}$ and $\mathrm{SU}(3)_{c}$ respectively (" $c$ " stands for color), where the electric charges of the above fermions where given in the introduction and the quarks transform as triplets under the color SU(3). We are left with weak interactions. While QED and QCD are 
parity conserving theories of charged particles and as such can be described in terms of Dirac spinors, we know that weak interactions violate parity. In order to describe weak interactions, we therefore expect to have to split the above spinors into left- and right-handed components $\nu_{L}, e_{L}, e_{R}, u_{L}, u_{R}, d_{L}, d_{R}$ (I did not include a right-handed neutrino component, so far unobserved). Indeed, it was known that weak interactions could be described in terms of the effective four-fermion interaction [4]

$$
\mathcal{L}_{\text {weak }}=4 \frac{G_{F}}{\sqrt{2}} j_{c}^{\mu} j_{c \mu}^{\dagger}, \quad \text { where } \quad G_{F}^{-1 / 2} \sim 250 \mathrm{GeV} \quad \text { and } \quad j_{c}^{\mu}=\overline{\nu_{L}} \gamma^{\mu} e_{L}+\overline{u_{L}} \gamma^{\mu} d_{L}
$$

only involves left-handed fields (thus breaking parity). We have written the charged current directly in terms of quarks, thus taking advantage of our present knowledge. The experimental determination of the Lorentz and chirality structure of the interaction above had been crucial for the discovery of the SM.

The coefficient of the interaction in eq. (8) has negative energy dimension. We are therefore dealing with a non-renormalizable interaction. The effect of such an interaction at energies $E \ll G_{F}^{-1 / 2}$ will be suppressed by powers of $E / G_{F}^{-1 / 2}$. That's why weak interactions are weak at low energy. At energies comparable to the scale $G_{F}^{-1 / 2}$ and above, however, the interaction above becomes stronger and stronger and the theory eventually becomes incalculable. This is signaled by the failure of the perturbative calculations of unitarity. The cross section for processes induced by the interaction in eq. (8) would in fact grow as $G_{F} E^{2}$ at high energy, instead of falling with $1 / E^{2}$, as predicted by unitarity. In order to be able to make sense of weak interactions at $E \sim G_{F}^{-1 / 2}$ and beyond, we need to replace the effective description in eq. (8) with a description in terms of renormalizable interactions. This is easily done. The effective interaction in eq. (8) can be generated by the exchange of an heavy field with mass $M$, as shown in Fig. 2 for the case of the leptonic interactions.

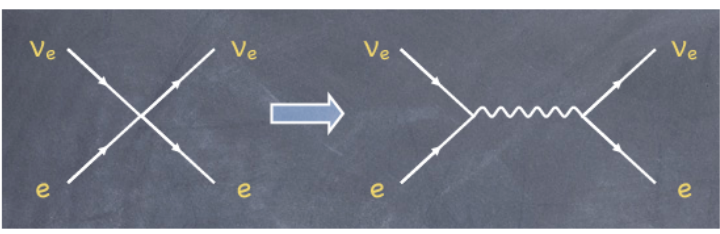

Figure 2: Weak interactions in terms of renormalizable physics.

When the energy $E$ involved in the process is smaller than $M$, the propagator $\left(E^{2}-M^{2}\right)^{-1}$ can be approximated by $-1 / M^{2}$, and one recovers the effective description of weak interactions. In other words, the wavelength of the process is too large to probe the detailed renormalizable structure of the interaction, which appears to be pointlike, as in the left hand side of Fig. 2. In this $E<M$ regime, the amplitude for the process in the Figure grows as $A \sim g^{2} / M^{2}$ and the cross section as $\sigma \sim g^{4}\left(E^{2} / M^{4}\right)$, where $g$ is the coupling involved in the renormalizable vertexes. On the other hand, when the energy becomes large, $E>M$, the propagator $\left(E^{2}-M^{2}\right)^{-1}$ can be approximated by $1 / E^{2}$, the amplitude goes as $A \sim g^{2} / E^{2}$ and the cross section as $\sigma \sim g^{4} / E^{2}$, in agreement with the unitarity bound. 
We now have to identify the degrees of freedom exchanged in Fig. 2 and their renormalizable interactions with the SM fermions. Here is where the determination of the Lorentz and chirality structure of the effective weak interactions turns crucial. The Lorentz structure indicates that the heavy particle exchanged is a (charged) vector, $W_{\mu}^{ \pm}$, and the chirality structure shows that it only couples to left-handed fermions. We can then write the fermion-vector interaction entering the Feynman diagram in Fig. 2 (and the corresponding quark one) as

$$
-\mathcal{L}_{W}=\frac{g}{\sqrt{2}} W_{\mu}^{+} \overline{\nu_{L}} \gamma^{\mu} e_{L}+\frac{g}{\sqrt{2}} W_{\mu}^{+} \overline{u_{L}} \gamma^{\mu} d_{L}+\text { h.c. }
$$

where the $\sqrt{2}$ is conventional. We can then express the Fermi constant $G_{F}$ in terms of the $W$ mass $M_{W}$ and coupling $g$ as $G_{F} / \sqrt{2}=g^{2} /\left(8 M_{W}^{2}\right)$. We have then learned two things. First, weak interactions involve a new vector field $W_{\mu}^{ \pm}$. As said, vectors should be described by means of a gauge theory. We should then find a gauge group $G_{\mathrm{SM}}$ and assign quantum numbers to the fermions in such a way that the interaction in eq. (9) be given by a gauge Lagrangian in the form in eq. (6). Second, we will have to understand how a vector boson, $W_{\mu}^{ \pm}$, can get massive, as we pointed out before that a mass term for a vector boson is not gauge invariant. This will require the spontaneous symmetry breaking of the gauge symmetry, which we will address in Section 4. In the meanwhile, in this Section, we will ignore the latter issue and determine the gauge structure of the SM.

In order to determine the gauge group $G_{\mathrm{SM}}$ and the fermion quantum numbers generating the gauge interactions in eq. (9), we need to compare eq. (9) with the general form of the vector-fermion interactions in eq. (7). Since eq. (7) involves real vectors, we first write $W_{\mu}^{ \pm}=\left(W_{\mu}^{1} \mp i W_{\mu}^{2}\right) / \sqrt{2}$ in terms of the real vectors $W_{\mu}^{1,2}$. The interactions in eq. (9) can then be written as $-\mathcal{L}_{W}=g \bar{L} \gamma^{\mu}\left(W_{\mu}^{1} T_{1}+W_{\mu}^{2} T_{2}\right) L+$ $g \bar{Q} \gamma^{\mu}\left(W_{\mu}^{1} T_{1}+W_{\mu}^{2} T_{2}\right) Q$, where $T_{1,2}=\sigma_{1,2} / 2$ and we have introduced the left handed lepton and quark doublets

$$
L \equiv\left(\begin{array}{c}
\nu_{L} \\
e_{L}
\end{array}\right) \quad Q \equiv\left(\begin{array}{c}
u_{L} \\
d_{L}
\end{array}\right) .
$$

It is now easy to compare with eq. (7) and identify two of the generators of the gauge theory we want to determine to be $T_{1}$ and $T_{2}$. Since the generators of a gauge theory form a Lie algebra and $\left[T_{1}, T_{2}\right]=i T_{3}$, with $T_{3}=\sigma_{3} / 2$, we conclude that $T_{3}$ is also a generator, to which a third gauge boson, $W_{\mu}^{3}$, must be associated. Note that $W_{\mu}^{3}$ cannot be identified with a photon or a gluon, the gauge bosons associated to QED and QCD (the couplings to fermions are different). The gauge principle therefore leads to the prediction of a new (neutral) interaction. The three generators above constitute the algebra of generators of $\mathrm{SU}(2)_{L}$, which must therefore be a factor of the gauge group, $G_{\mathrm{SM}} \supseteq \mathrm{SU}(2)_{L}$. The index $L$ refers to the fact that $\mathrm{SU}(2)_{L}$ only 
acts on the left handed components of the fields. The latter organize themselves in doublets of $\mathrm{SU}(2)_{L}$, as in eq. (10), whereas the right handed components should be $\mathrm{SU}(2)_{R}$ singlets, as they do not appear in $\mathcal{L}_{W}$.

Since we know that electromagnetic and strong interactions are also described by a gauge theory, the gauge group $G_{\mathrm{SM}}$ should also include the corresponding generators. Strong interactions can be accounted for by a $\mathrm{SU}(3)_{c}$ factor commuting with the rest of the SM group, under which $u_{L}, u_{R}, d_{L}, d_{R}$ all transform as triplets (they carry a "color" index). We do not need to discuss them here. Let us instead concentrate on the electromagnetic interactions. They are associated to a electric charge generator $Q$ (not to be confused with the quark doublet in eq. (10)), whose value on the fermions gives their electric charges (by convention in units of $e$ ). As a linear combination of generators is still a generator, the combination $Y \equiv Q-T_{3}$ should also be a generator, which is called "hypercharge". The interest of $Y$ is that it turns out to commute with the $\mathrm{SU}(2)_{L}$ generators. The hypercharges of the fermions can be computed in terms of their electric and $T_{3}$ charges. One then finds that the hypercharges of the two lepton doublet components, $\nu_{L}$ and $e_{L}$, coincide: $Y\left(\nu_{L}\right)=Y\left(e_{L}\right)=-1 / 2$. Therefore, the hypercharge and $\mathrm{SU}(2)_{L}$ generators commute on the lepton doublet $L$. But then they should also commute on the quark doublet $Q$. This means that the hypercharges of $u_{L}$ and $d_{L}$ should also coincide, if what we are doing makes sense. Let us then cross our fingers and compute those hypercharge. For $u_{L}$ we have $Y\left(u_{L}\right)=Q\left(u_{L}\right)-T_{3}\left(u_{L}\right)=$ $2 / 3-1 / 2=1 / 6$ and for $d_{L} Y\left(d_{L}\right)=Q\left(d_{L}\right)-T_{3}\left(d_{L}\right)=-1 / 3-(-1 / 2)=1 / 6$. This means that we are one the right track (unfortunately, about 40 years too late for the Nobel prize). The generator $Y$ is therefore associated to a $\mathrm{U}(1)_{Y}$ subgroup of $G_{\mathrm{SM}}$ that commutes with the rest of the group. All in all, this model building exercise identifies the SM gauge group to be

$$
G_{\mathrm{SM}}=\mathrm{SU}(3)_{c} \times \mathrm{SU}(2)_{L} \times \mathrm{U}(1)_{Y}
$$

where the last two factors constitute the electroweak group. The weak and electromagnetic interactions are described by this subgroup, with the electromagnetic interactions associated with a combination of $\mathrm{SU}(2)_{L}$ and $\mathrm{U}(1)_{Y}$ generators, $Q=Y+T_{3}$. The quantum numbers of the fermions with respect to the three factors of the group are summarized in Table 1. The first two columns show the transformation properties under $\mathrm{SU}(3)_{c}$ and $\mathrm{SU}(2)_{L}$, while the last columns shows the hypercharge of each field. The quantum numbers are the same for the three families, which goes under the name of family replication. We do not have a compelling explanation for such a replication. The values of the hypercharges for the right-handed fermions are given by $Y=Q$, as the $\mathrm{SU}(2)_{L}$ generators vanish on the right-handed fermions. Table 1 fully specifies the SM gauge interactions of the SM fermions. We will analyze them in detail in Section 6. 


\begin{tabular}{|c|ccc|}
\hline & $\mathrm{SU}(3)_{c}$ & $\mathrm{SU}(2)_{L}$ & $\mathrm{U}(1)_{Y}$ \\
\hline$L$ & 1 & 2 & $-1 / 2$ \\
$e_{R}$ & 1 & 1 & -1 \\
$Q$ & 3 & 2 & $1 / 6$ \\
$u_{R}$ & 3 & 1 & $2 / 3$ \\
$d_{R}$ & 3 & 1 & $-1 / 3$ \\
\hline
\end{tabular}

Table 1: Gauge quantum numbers of the SM fermions (one family).
Before closing this Section, let us discuss anomalies. The quantum numbers in Table 1 have quite a peculiar pattern. What is (pleasingly) surprising about it is that they satisfy the gauge anomaly cancellation conditions. Anomalies arise when a symmetry of the Lagrangian is not preserved by quantum corrections so that the corresponding current is conserved at the classical level but not at the quantum level. In some case, anomalous symmetries are not a problem,

they are actually welcome. This is the case for example of the (global) chiral symmetry of QCD, or of scale invariance, which is broken at the quantum level by the renormalization scale, leading to the anomalous dimensions of fields. In the case of gauge symmetries, however, anomalies should be avoided in order not to make the theory inconsistent. There is a simple condition that needs to be verified in order for gauge anomalies to vanish. The presence of gauge anomalies depends on how the gauge group generators act on the left-handed fermions $\left(\psi_{i L}\right.$ and $\left.\psi_{i R}^{*}\right)$. Let $T_{a}^{L}$ give the action of the generators on the left-handed fermions. Then the gauge symmetry survives quantum corrections iff $0=T_{a b c} \equiv \operatorname{tr}\left(T_{a}^{L}\left\{T_{b}^{L}, T_{c}^{L}\right\}\right)$ for each choice of generators. As a consequence of this condition, a gauge symmetry acting in the same way on left and right chirality fermions (as QED, QCD) turns out to be automatically non-anomalous. There is no guarantee, however, that a chiral theory such as the SM be non-anomalous. In order to check whether this is the case we need to compute $T_{a b c}$ for a combination of any three SM generators. It is a useful exercise to verify that $T_{a b c}$ indeed always vanishes. This is a highly non-trivial property depending on the fact that i) $\mathrm{SU}(3)_{c}$ acts in the same way on left and right chirality fermions, ii) the traces of $\mathrm{SU}(2)$ and $\mathrm{SU}(3)$ generators vanish, iii) three non-trivial relations hold among the hypercharge quantum numbers: $2 Y_{Q}-Y_{u_{R}}-Y_{d_{R}}=0$, $Y_{L}+3 Y_{Q}=0$, and $2 Y_{L}^{3}+6 Y_{Q}^{3}-3 Y_{u_{R}}^{3}-3 Y_{d_{R}}^{3}-Y_{e_{R}}^{3}=0$. Moreover, the relation $2 Y_{L}+6 Y_{Q}-3 Y_{u_{R}}-3 Y_{d_{R}}-Y_{e_{R}}=0$ accounts for the vanishing of another type of anomaly, the gravitational one. The fact that the SM is anomaly free is reassuring. Of course it would be nice to understand whether there is a reason, besides the cancellation of anomaly itself, why the values of the hypercharge we measure happen to satisfy the anomaly cancellation condition, or, more generally, if there is a reason for the peculiar pattern of gauge quantum numbers in Table 1. Grand-unification theories may answer such questions. 


\section{Spontaneous symmetry breaking (SSB)}

In order to proceed further we need to address the puzzle of vector boson masses. The $W_{\mu}^{ \pm}$vector bosons leading to the effective interaction in eq. (8) have a mass of about $80 \mathrm{GeV}$. On the other hand, the gauge symmetry prevents a mass term for the gauge vectors. The gauge symmetry must then be somehow broken, in order for the gauge vectors to acquire a mass. This can be done through the mechanism of spontaneous symmetry breaking, which we discuss in this Section. Before illustrating it, let us observe that the vector boson masses are not the only problem. The SM fermions would also forced to be massless in the presence of an exact $\mathrm{SU}(2)_{L} \times \mathrm{U}(1)_{Y}$ symmetry. One can in fact verify that no gauge invariant fermion mass term can be written, given the quantum numbers in Table 1 . The most general mass term couples two left-handed fermions in a combination that, as all the terms in the Lagrangian, must be invariant under the gauge symmetry. There are three possible types of mass terms that can be formed: $\psi_{L} \psi_{L}, \psi_{R}^{*} \psi_{L}, \psi_{R}^{*} \psi_{R}^{*}$. It is easy to verify that no such combination of left or right fermions in Table 1 can be gauge invariant. This is due to the SM gauge symmetry being chiral even when restricted to an arbitrary subset of fermions ${ }^{3}$. Spontaneous breaking of the gauge symmetry is then needed in order to account for the SM spectrum of both vector bosons and fermions. In the following, I will illustrate the main features of spontaneous symmetry breaking of global and gauge symmetries (Higgs mechanism). In the next Section we will see how this applies to the SM.

Spontaneous symmetry breaking (SSB) is interesting and elegant because no explicit breaking of the symmetry is introduced. The equations of the dynamics are exactly symmetric, but they admit solutions that are not. In particular, one has SSB when the ground state of the system is not symmetric. It is then the system itself that "spontaneously" breaks the symmetry. In the context of QFT, SSB is characterized by i) the Lagrangian being invariant under the symmetry, ii) the currents associated to the symmetry being conserved, also at the quantum level, iii) the vacuum (ground state) of the theory being not invariant under the symmetry, iv) the spectrum being not invariant. Its features are i) it allows a consistent breaking of gauge symmetries, in particular a consistent quantization of massive vectors; ii) it turns out to be realized in nature both in the case of global and gauge symmetries.

\footnotetext{
${ }^{3}$ From the point of view of the physics at the electroweak scale, the chiral structure of the SM is a puzzle. From the point of view of physics at the Planck scale, however, such structure is welcome because it explains why the SM fermions are so light with respect to the Planck scale: they are prevented from getting a mass term until the electroweak symmetry is broken, at a much lower scale. One can take this argument a step further and argue that this is the reason why the SM fermions are fully chiral: because if they were not there would be no reason why they should not be much heavier. Chiral fermions may just be the only ones surviving at low energy because of their very chiral structure.
} 


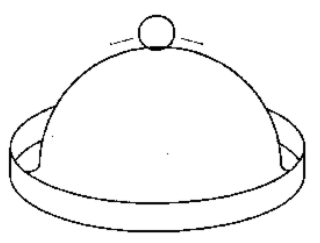

Figure 3: An example of SSB in classical mechanics.

There are several classic examples of SSB. In classical mechanics one can consider a ball forced to live on the symmetric (under rotations around the central axis) surface in Fig. 3 subject to a gravitational field pointing downwards. The equations of the dynamics are symmetric. The central point, where the ball is in the Figure, is also symmetric, but unstable. In order to live in a stable ground state, the system should choose a position a the bottom of the surface, thus spontaneously breaking the rotational symmetry. In quantum mechanics, one can consider a rotationally invariant system of coupled spins (a ferromagnet). The minimum energy state is reached when the spins are aligned. In order to live in the ground state, therefore, the system must choose a common direction for the spins, thus spontaneously breaking rotational invariance. In QFT, a qualitative difference arises, as the number of degrees of freedom is not finite anymore and a quantum superposition of degenerate vacua is not allowed: different ground states are not described within the same Hilbert space.

We now discuss in more detail SSB in QFT. We are interested to the spontaneous breaking of gauge invariance, but we need to discuss the spontaneous breaking of global symmetries first. Let us call $\Omega$ the ground state of our QFT. SSB arises iff the vacuum expectation value (vev) of the fields in the theory, $\langle\Phi\rangle \equiv\langle\Omega|\Phi(x)| \Omega\rangle$, is not invariant (therefore not vanishing) under the symmetry $(\langle\Phi\rangle$ is invariant if $\Omega$ is). As we do not want to break Poincaré invariance, only scalars can get a non vanishing vev and $\langle\Phi(x)\rangle$ does not depend on the space-time coordinate $x$. Given a Lagrangian, the value of the vev of the scalar fields can be easily obtained by minimizing the effective scalar potential, i.e. the scalar potential including the so-called one-particle irreducible quantum corrections. It is often possible to neglect quantum corrections and just consider the minimization of the classical potential, which is what we will do in the following. A simple explicit example of SSB in QFT can be obtained in the theory of a complex scalar field $\phi$ with Lagrangian

$$
\mathcal{L}=\left(\partial_{\mu} \phi\right)^{\dagger}\left(\partial^{\mu} \phi\right)-V\left(\phi^{\dagger} \phi\right), \quad V\left(\phi^{\dagger} \phi\right)=\mu^{2} \phi^{\dagger} \phi+\frac{\lambda}{2}\left(\phi^{\dagger} \phi\right)^{2}
$$

The Lagrangian is symmetric under a global $\mathrm{U}(1)$ transformation $\phi(x) \rightarrow e^{-i \alpha} \phi(x)$. The parameter $\lambda$ must be non-negative in order for the potential to be bounded from below. We will take it to be strictly positive. On the other hand, the parameter $\mu^{2}$ can have both signs (despite it is written as a square to stress that it has the dimension of a squared mass). The shape of the potential $V$ and the structure of the ground state crucially depends on that sign, as shown in Fig. 4 . If $\mu^{2}<0$, the minimum of the potential corresponds to $\langle\phi\rangle=v e^{i \theta}$, where 


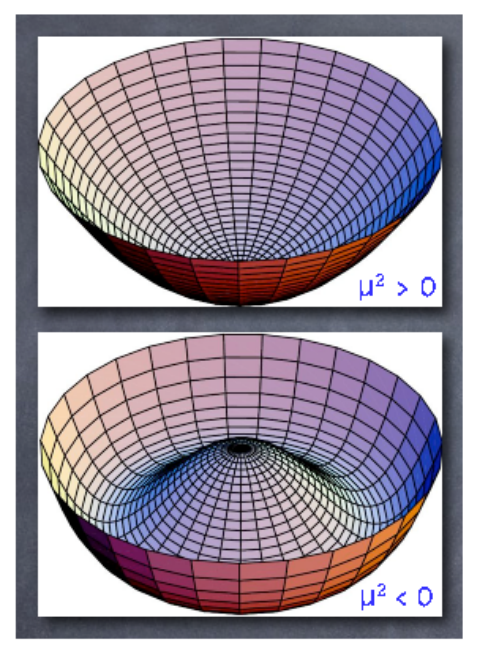

Figure 4: Graphical representation of the potential in (13).

$v^{2}=\left|\mu^{2}\right| / \lambda$ and $\theta$ parametrizes the position of $\phi$ in the circle with radius $v$ of degenerate minima. The system chooses an arbitrary value of $\theta$, thus spontaneously breaking the $\mathrm{U}(1)$ symmetry. With no loss of generality, we can assume $\theta=0$.

In view of the shape of the potential, it is convenient to parametrize the field as $\phi(x)=r(x) e^{i g(x)}$, where $g(x)$ parametrizes the "flat directions" along which the potential is constant. As $V$ does not depend on $g(x)$, the corresponding real degree of freedom is massless and only has derivative interactions. Such massless degrees of freedom always arise in the presence of SSB in QFT. They are called "Goldstone" bosons. One can also use a linear parametrization of the Goldstone boson by expanding $\phi(x)=v+\phi^{\prime}(x)=v+(h(x)+i G(x)) / \sqrt{2} . G(x)$ can be considered as the linearization of $v g(x)$. In terms of $h$ and $G$, the potential is

$$
V=\frac{\lambda}{8}\left(h^{2}+G^{2}\right)^{2}+\frac{\lambda}{\sqrt{2}} v h\left(h^{2}+G^{2}\right)+\left|\mu^{2}\right| h^{2}+\text { const. }
$$

We can again verify that $G$ is massless, whereas the physical degree of freedom $h$ acquires a mass proportional to the symmetry breaking scale $v$ and to its self-coupling, $m_{h}^{2}=2\left|\mu^{2}\right|=2 \lambda v^{2}$. The two parameters $\mu^{2}, \lambda$ in the potential $V$ can be traded for $v$ and $m_{h}^{2}$.

The discussion above can be generalized to the case of a generic continuous global symmetry group $G$ and a generic scalar field content. Here are the main features of the generalization. Let us call $H$ the subgroup of $G$ that is not broken by the vev of the scalar fields (under which the vev of the scalar fields is invariant). We can correspondingly divide the generators of $G$ into two sets: the unbroken ones, which annihilate the vacuum, and the broken ones, the orthogonal set. According to the Goldstone theorem [5] each broken generator in $G / H$ is associated to an independent massless scalar (Goldstone boson), carrying the same quantum numbers as the generators.

The prototypical example of spontaneously broken global symmetry in QFT is the chiral symmetry of QCD with two quarks. In the limit in which the up and down quark masses coincide the QCD Lagrangian (not including electromagnetic interactions) for $u$ and $d$ is invariant under independent unitary transformations of the left handed and right handed components of the $u$ and $d$ fields, corresponding to the symmetry group $\mathrm{U}(2)_{L} \times \mathrm{U}(2)_{R}=\mathrm{SU}(2)_{L} \times \mathrm{U}(1)_{L} \times \mathrm{SU}(2)_{R} \times \mathrm{U}(1)_{R}$. Let us call $j_{L}^{\mu a}, j_{L}^{\mu}, j_{R}^{\mu a}, j_{R}^{\mu}$ the currents associated to the four group factors. Then $j_{V}^{\mu}=j_{R}^{\mu}+j_{L}^{\mu}$ is the conserved current associated to Baryon number and $j_{A}^{\mu a}=j_{R}^{\mu a}+j_{L}^{\mu a}$ are the conserved isospin 
currents. What about the "axial" currents $j_{A}^{\mu}$ and $j_{A}^{\mu a}$ ? The first one, $j_{A}^{\mu}$, turns out to be broken by quantum corrections. The corresponding symmetry is anomalous. The second current, $j_{A}^{\mu a}$, turns out to be conserved, on the other hand. Therefore, it either corresponds to a symmetry or it is spontaneously broken. We do not have evidence for $j_{A}^{\mu a}$ to correspond to a symmetry. If it did, particles would organize themselves in multiplets with same spin, Baryon number, parity and approximately (in the real world in which $m_{u} \neq m_{d}$, electromagnetic interactions exist, and the chiral symmetry $\mathrm{SU}(2)_{L} \times \mathrm{SU}(2)_{R}$ is only approximate) same mass, which we do not observe. On the other hand, we do have evidence for the symmetry to be spontaneously broken. If that is the case, we should observe three light (not exactly massless in the real world) pseudo- Goldstone bosons with zero Baryon number, spin, negative parity and isospin 1 , as the corresponding broken generators. The lightest hadrons, the pions, have indeed all those properties. They are therefore considered to be the pseudo- Goldstone bosons arising because of the spontaneous breaking $\mathrm{SU}(2)_{L} \times \mathrm{SU}(2)_{R} \rightarrow \mathrm{SU}(2)_{V}$ of the approximate chiral symmetry of the light quark QCD Lagrangian.

We have discussed so far the spontaneous breaking of a global symmetry. We now discuss the case we are more directly interested in, the spontaneous breaking of gauge invariance. The main feature of such a phenomenon is that the gauge vector associated to each broken generator gets a longitudinal component and a mass. The additional longitudinal degree of freedom is provided by the Goldstone boson associated to the broken generator, which gets "eaten up" by the vector. This is just what we need in order to describe the massive vector boson we observe in nature, the $W^{ \pm}$and the $Z$, in a theoretically consistent way.

In order to see how this works, let us consider the complex scalar field again and promote the global U(1) symmetry to a gauge symmetry. Our gauge theory machinery gives

$$
\mathcal{L}_{\text {gauge }}=-\frac{1}{4} F_{\mu \nu} F^{\mu \nu}+\left(D_{\mu} \phi\right)^{\dagger}\left(D^{\mu} \phi\right)-V\left(\phi^{\dagger} \phi\right)+\text { gauge fixing, } \quad D_{\mu}=\partial_{\mu}+i g A_{\mu} .
$$

Let us break $\mathrm{U}(1)$ spontaneously by taking $\mu^{2}<0$. The complex field develops a vev $\langle\phi\rangle=v$, as before. Correspondingly, a mass term $M^{2}=2 g^{2} v^{2}$ is generated for the vector boson, proportional to the symmetry breaking scale and to its (gauge) coupling to $\phi$, as can be seen from $\left(D_{\mu} \phi\right)^{\dagger}\left(D^{\mu} \phi\right)=\left(\partial_{\mu} \phi^{\prime}\right)^{\dagger}\left(\partial^{\mu} \phi^{\prime}\right)+M^{2} A^{\mu} A_{\mu} / 2+\ldots$ The Goldstone boson gets eaten by the vector boson, of which it becomes the longitudinal component. This can be seen by parametrizing $\phi(x)$ in terms of $r(x)$ and $g(x)$ as above and by noticing that

$$
\left\{\begin{array} { l } 
{ \phi ( x ) = r ( x ) e ^ { i g ( x ) } } \\
{ A _ { \mu } ( x ) }
\end{array} \quad \text { is equivalent to } \left\{\begin{array}{l}
\phi(x)=r(x) \\
A_{\mu}(x)-\frac{1}{g} \partial_{\mu} g(x)
\end{array},\right.\right.
$$

as the two configurations are related by a gauge transformation. We can therefore choose a "unitary" gauge in which the field $\phi(x)$ is real, as on the right side of eq. (15), 
and does not contain $g(x)$, which can be recognized as the longitudinal component of $A_{\mu}(x)$. This can be generalized to the case of a generic continuous global symmetry group $G$ and a generic scalar field content. In the general case, as mentioned, the gauge vector associated to each broken generator gets a mass by absorbing the corresponding Goldstone boson. This is what goes under the name of "Higgs" mechanism. In the context of gauge theories, unlike the case of global symmetries, the Goldstone bosons do not correspond to physical scalar degrees of freedom. The scalar fields spontaneously breaking the gauge symmetry are called Higgs fields.

Before closing this Section we mention that global and gauge symmetries can be spontaneously broken without the need of scalar fields. The role of the vev of the scalar field is then played by the condensate of a fermion bilinear that arises dynamically as a consequence of new strong interactions. This is how chiral symmetry breaking in QCD is thought to arise. While it is not excluded that such dynamical symmetry breaking mechanism plays a role in the breaking of the electroweak symmetry, such a possibility is at present disfavored. We will not further consider it in these lectures.

\section{The SM Higgs and flavour}

We are now ready to go back to the SM and discuss its spontaneous breaking. The existence of fermion and vector boson masses is evidence that the SM gauge invariance should be spontaneously broken. We know on the other hand that strong and electromagnetic interactions are not broken as, for example, the electric charge is conserved and the photon is massless. Therefore, the SSB of the SM should preserve $\mathrm{SU}(3)_{c} \times \mathrm{U}(1)_{\mathrm{em}}$ as an unbroken subgroup.

In order to spontaneously break the SM, we should introduce a scalar field, the Higgs, developing a vev and specify its quantum numbers under the SM gauge group. Such quantum numbers are dictated by the need of the fermions to get a mass term, as we now see.

Let us consider the electron mass term, which has the form $m\left(\overline{e_{R}} e_{L}+\right.$ h.c. $)$. As the left component $e_{L}$ is contained in the lepton doublet $L$, that interaction should originate from a SM invariant interaction involving the bilinear $\overline{e_{R}} L$. Now, such a bilinear is not invariant under $G_{\mathrm{SM}}$. It transforms as a doublet with $Y=1 / 2$ under $\mathrm{SU}(2)_{L} \times \mathrm{U}(1)_{Y}$ (and is of course invariant under $\left.\mathrm{SU}(3)_{c}\right)$. That is why the electron mass is not allowed by the gauge symmetry. However, $\overline{e_{R}} L$ can be part of a gauge invariant renormalizable interaction involving an additional field with appropriate quantum numbers. The only possibility is the Yukawa interaction $\lambda \overline{e_{R}} L H^{*}$ with a complex doublet scalar field $H$ (conventionally taken as the conjugated of the field appearing in the interaction), the Higgs field. $H$ is a doublet with $Y=1 / 2$ under $\mathrm{SU}(2)_{L} \times \mathrm{U}(1)_{Y}$ and is a $\mathrm{SU}(3)_{c}$ singlet. Its two components contract with the two components in $L$. A mass term for the electron can now be generated if $H$ gets a vev, 
once the value of the vev is substituted to the field in the Yukawa interaction above. It turns out that one Higgs doublet $H$ is enough to give rise to the mass of all the SM fermions. Let us in fact write the most general Yukawa Lagrangian involving the Higgs field. Such a Lagrangian is given by

$$
-\mathcal{L}_{Y}=\lambda_{i j}^{E} \overline{e_{i R}} L_{j} H^{*}+\lambda_{i j}^{D} \overline{d_{i R}} Q_{j} H^{*}+\lambda_{i j}^{U} \overline{u_{i R}} Q_{j} H+\text { h.c. }
$$

where we have included all the three SM families through the family indices $i, j=$ $1,2,3$. As a consequence of this family structure, each of the three Yukawa couplings in eq. (16) is a generic $3 \times 3$ complex matrix. Once the Higgs gets a vev, all the $\mathrm{SM}$ fermions get a mass proportional to their Yukawa couplings. SU(2) and SU(3) contractions have been understood in eq. (16). The SU(2) invariant contraction of the doublet indices of $Q$ and $H$ in the up quark Yukawa interaction is obtained by means of the $2 \times 2$ antisymmetric tensor $\epsilon_{a b}$ as $Q H=Q_{a} \epsilon_{a b} H_{b}\left(\epsilon_{12}=1\right)$. The Lagrangian $\mathcal{L}_{Y}$ is the origin of the flavour structure of the SM. It is because of that Lagrangian that we can tell for example an electron from a muon. The gauge Lagrangian, in fact, does not make any difference between them.

In order to fully specify the SM Lagrangian we only miss the Higgs Lagrangian. In the same spirit used for the Yukawa Lagrangian, we can simply write the most general renormalizable Lagrangian involving the Higgs field. That turns out to be

$$
\mathcal{L}_{H}=\left(D_{\mu} H\right)^{\dagger}\left(D^{\mu} H\right)-V, \quad V=\mu^{2} H^{\dagger} H+\frac{\lambda_{H}}{2}\left(H^{\dagger} H\right)^{2}
$$

As in the SSB example with a single complex scalar field, we take $\lambda_{H}>0$ and $\mu^{2}<0$ in order to obtain a stable, symmetry breaking potential.

This completes the definition of the SM. We have chosen the gauge group as in eq. (11); we have specified the fermion and scalar content (the three replications of the fermions in Table 1 and the Higgs doublet); their quantum numbers under the SM gauge group (Table 1 and the Higgs assignment above); we have also specified the most general renormalizable globally symmetric Lagrangian for the above fields (the kinetic terms of all the fermions $+\mathcal{L}_{Y}+\mathcal{L}_{H}$ ). The gauge theory machinery then allows to specify the full Lagrangian as in eq. (6). We will analyze the Higgs Lagrangian, together with the rest of the SM Lagrangian, in the next Section.

\section{Analysis of the SM Lagrangian}

Having defined and motivated the SM, let us know analyze the SM Lagrangian and spell out some phenomenological implications. Let us start from where we stopped, the Higgs sector. 
In the minimum of its potential, the Higgs doublet develops a vev. With no loss of generality, such a vev can be written as

$$
\langle H\rangle=\left(\begin{array}{l}
0 \\
v
\end{array}\right), \quad \text { with } \quad v>0 \text { and } v^{2}=\frac{\left|\mu^{2}\right|}{\lambda_{H}} \approx(174 \mathrm{GeV})^{2} .
$$

Any other form of the Higgs vev, in fact, is equivalent to that in eq. (18), up to a $\mathrm{SU}(2)_{L} \times \mathrm{U}(1)_{Y}$ gauge transformation. The scale $v \approx 174 \mathrm{GeV}$ is called the electroweak symmetry breaking scale, or electroweak scale. Let us identify the unbroken part of the SM group, or equivalently the generators that annihilate the Higgs vev. Since the Higgs does not feel strong interactions, the latter are certainly unbroken. Let us therefore concentrate on the electroweak group $\mathrm{SU}(2)_{L} \times \mathrm{U}(1)_{Y}$. Its generic generator can be written as $T=a Y+b_{a} T_{a}$, with $a, b_{a}$ real. When acting on the Higgs, $T_{a}=\sigma_{a} / 2$ and $Y=1 / 2$. Therefore $T\langle H\rangle=(v / 2)\left(b_{1}-i b_{2}, a-b_{3}\right)^{T}$ and the unbroken generators, for which $T\langle H\rangle=0$, are those for which $b_{1}=b_{2}=0$ and $a=b_{3}$. There is then only one (up to normalization) electroweak generator unbroken by the Higgs vev, given by $T_{3}+Y=Q$. The electric charge is unbroken, as wished. Note that the latter can be considered as a prediction, as the Higgs quantum numbers, determining the unbroken generators, were fixed by independent considerations (obtaining a mass for the electron). Out of the 4 generators of the electroweak group only one is unbroken, which means that 3 are broken. We then expect 3 vector bosons to acquire a mass and 3 Higgs real degree of freedom (the Goldstones) to be eaten up by them. Out of the 4 real (2 complex) Higgs degrees of freedom, only one then correspond to a physical scalar, the Higgs boson. In order to identify the Goldstone (and thus the physical) degrees of freedom we can use a general property of the Goldstone bosons: they correspond to displacements from the vev along the flat directions of the potential. We can move along the flat directions of the potential by performing $G_{\mathrm{SM}}$ transformation (which leave the potential invariant) along an arbitrary set of broken generators. From $\delta\langle H\rangle=i \epsilon G_{a} T_{a}\langle H\rangle=\epsilon(v / 2)\left(i G_{1}+G_{2},-i G_{3}\right)^{T}$ we see that we can write the Higgs doublet in terms of the Goldstone components $G^{ \pm}=\left(G_{1} \mp i G_{2}\right) / \sqrt{2}$, $G_{0}=-G_{3}$ and the physical component $h$ as

$$
H=\left(\begin{array}{c}
i G^{+} \\
v+\frac{h+i G^{0}}{\sqrt{2}}
\end{array}\right)
$$

With respect to the CP transformation under which $H \rightarrow H^{*}, h$ is even and the Goldstones are odd. We can write the Higgs potential in the unitary gauge in which the Goldstones are removed from the Higgs fields and incorporated in the corresponding vector bosons as follows:

$$
V(h)=V(H)_{G=0}=\frac{m_{h}^{2}}{2} h^{2}+\frac{\lambda_{H}}{\sqrt{2}} v h^{3}+\frac{\lambda_{H}}{8} h^{4}+\text { const },
$$


where the Higgs mass is given by $m_{h}^{2}=2|\mu|^{2}=2 \lambda_{H} v^{2}$ and is proportional to the electroweak symmetry breaking scale and to the Higgs self-coupling $\lambda_{H}$.

While the electroweak scale is known from the measurement of the Fermi constant $G_{F}$, as we will see, the Higgs mass (or equivalently the Higgs coupling) is at the moment an unknown parameter. We have however three different constraints on it.

The first is a theoretical constraint: in order to avoid a strong coupling regime, the Higgs mass should be lighter than about a TeV. If the Higgs mass was heavier than that, the theory would become strongly interacting before the Higgs could be produced. This is not a priori excluded. However, keeping the theory perturbative allows a quantitative extrapolation to higher energies. Moreover, there are constraints from precision tests on generic effects of strong interactions at a scale as low as a $\mathrm{TeV}$. The onset of a perturbative regime can be seen as follows. One can compute the amplitude $A\left(W_{L} W_{L} \rightarrow W_{L} W_{L}\right)$ for the scattering of the longitudinal component of the $W$ boson. The latter are nothing but the Goldstone bosons originally sitting in the Higgs doublet together with the physical Higgs boson whose mass we are trying to constrain. The expansion in partial waves gives $A=\sum_{l} a_{l} A_{l}$, where $a_{l}$ are partial wave amplitudes. The $s$-wave amplitude is bound by unitarity to be $\left|a_{0}\right| \leq 1$. If the physical Higgs is not taken into account, a tree level calculation, involving the gauge boson self couplings, gives $a_{0} \sim s /\left(16 \pi v^{2}\right)$, where $s$ is the center of mass squared energy. The unitarity bound would then be saturated for $s \approx(1.2 \mathrm{TeV})^{2}$, unless such a bad behaviour is cancelled by the diagrams involving the exchange of a Higgs lighter than $1.2 \mathrm{TeV}$. Since we know that unitarity is not violated, the apparent violation must be due to the failing of the tree level approximation, signaling in turn a strongly interacting regime where higher order perturbative corrections are as large as the lower order contributions.

A stronger, second constraint can be obtained by assuming that the SM holds and is stable and perturbative up to a scale $\Lambda$. This argument uses the fact that the Higgs coupling $\lambda_{H}$, as all the couplings in the Lagrangian, depends on the energy scale of the process in which it is involved. Under the assumption that the SM holds up to the scale $\Lambda$, the value of the Higgs coupling at any scale up to $\Lambda$ can be calculated as a function of the value at the electroweak scale, i.e. of the Higgs mass. It turns out that a too large value of the Higgs mass would give rise to a steep raise of $\lambda_{H}$ with the energy, leading to a Landau-pole, i.e. a non-perturbative regime, before the scale $\Lambda$, thus contradicting the initial hypotheses. We obtain this way an upper limit on the Higgs mass as a function of the scale $\Lambda$. On the other hand, a too small value of the Higgs mass would make $\lambda_{H}$ negative before $\Lambda$, thus giving rise to an instability. The Higgs potential would in fact become deeply negative for values of the Higgs field larger than the scale at which $\lambda_{H}$ becomes negative. The electroweak scale minimum we need, eq. (18), would therefore be at best metastable (in which case, assuming we happen to live in such a metastable vacuum, its lifetime should be larger than the life of the universe). All this leads to a lower limit on the Higgs mass as a function 
of the scale $\Lambda$. All in all, one gets a window for the Higgs mass $m_{h}$ as a function of the scale $\Lambda$. Such a window is shown in Fig. 5 for $10^{3} \mathrm{GeV}<\Lambda<10^{19} \mathrm{GeV}$ [6].

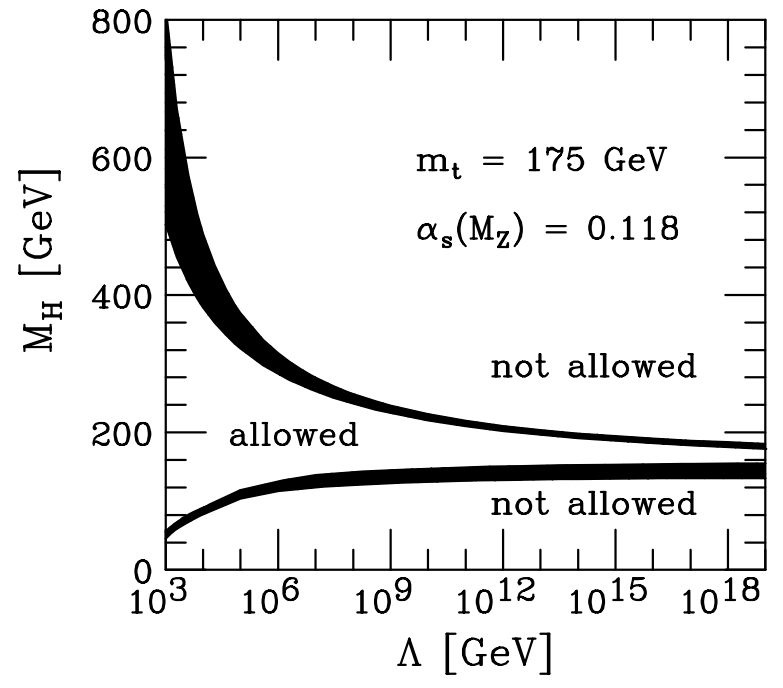

Figure 5: Allowed window for the Higgs mass as a function of the scale $\Lambda$ up to which the SM is assumed to hold and be stable and perturbative.

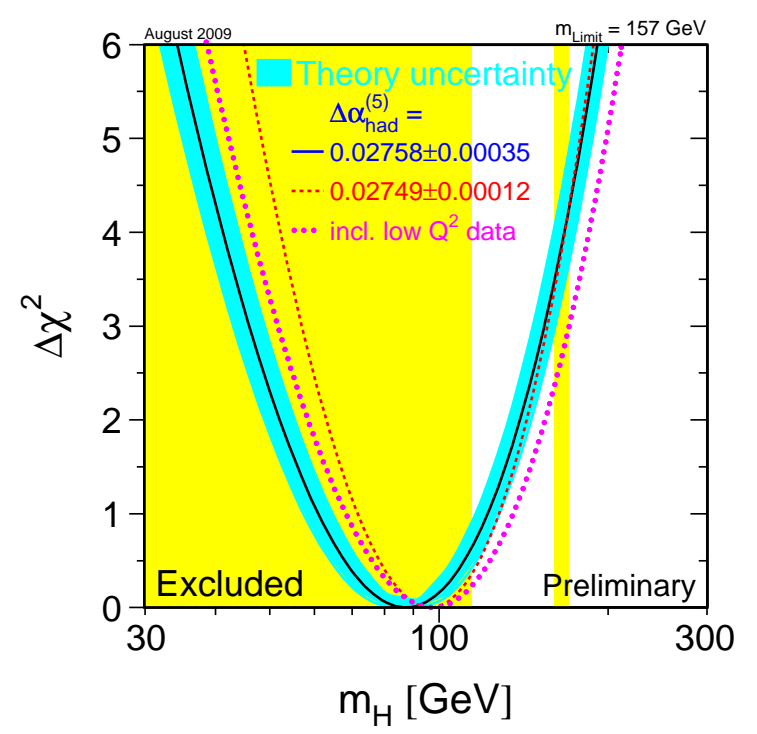

Figure 6: Direct (yellow exclusion regions) and indirect (blue band $\chi^{2}$ ) experimental bounds on the Higgs mass [7].
Let us now come to the experimental constraints on the Higgs mass. The direct experimental limit from LEP is $m_{h}>114 \mathrm{GeV}$ at $95 \% \mathrm{CL}$. Also, Tevatron has recently excluded the (160170) $\mathrm{GeV}$ window. On top of those, there are indirect experimental bounds. The Higgs mass enters in fact (logarithmically) through loop corrections a number of observables that have been precisely measured at colliders, LEP in particular. A global fit of such precision observables as a function of the Higgs mass actually favors an Higgs mass in the region excluded by LEP, but values above the LEP bound do not give a bad fit. All in all, the fit favours a relatively light Higgs, $m_{h}<$ $163 \mathrm{GeV}$ at $95 \% \mathrm{CL}$. The results of the fit are summarized in the "blue band plot" shown in Fig. 6 [7]. The fit could be modified in the presence of new physics.

The existence of a scalar (Higgs) mass parameter gives rise to the socalled naturalness problem of the SM, whose solution has represented in the last decades one of the main guidelines for the theoretical quest for new physics beyond the SM. This issue will be addressed in the lectures by Alexei Gladyshev.

Let us now come to the analysis of the gauge sector of the SM. If the Higgs sector of the SM is the least known part of the SM (we do not even know if the Higgs really exists), the gauge sector is on the contrary the best known sector. 
The gauge interactions of SM fermions have been tested with an accuracy up to the $\% o$ level, which is enough to probe the gauge sector at the loop level (perturbative loop corrections are typically of order $1 /(4 \pi)^{2} \sim \mathcal{O}(\%)$ ). The fermion gauge interactions can be obtained from the covariant derivative. Let us consider electroweak interactions only, as strong interactions will be discussed elsewhere. The covariant derivative then assumes the form $D_{\mu}=\partial_{\mu}+i g W_{\mu}^{a} T_{a}+i g^{\prime} B_{\mu} Y$, where $B_{\mu}$ is the gauge vector associated to the hypercharge generator. The covariant derivative involves two independent gauge couplings, $g$ and $g^{\prime}$, corresponding to the two irreducible factors of the electroweak group, $\mathrm{SU}(2)_{L}$ and $\mathrm{U}(1)_{Y}$. The explicit form of the generators $T_{a}$ and $Y$ depends on the quantum numbers of the field on which they act. For example, when acting on the Higgs field, $D_{\mu}=\partial_{\mu}+i g W_{\mu}^{a}\left(\sigma_{a} / 2\right)+i g^{\prime} B_{\mu}(1 / 2)$. The vector boson masses arise from the $\left(D_{\mu}\langle H\rangle\right)^{*}\left(D^{\mu}\langle H\rangle\right)$ term in the Higgs Lagrangian. To compute the vector boson masses, we observe that

$$
D_{\mu}\langle H\rangle=\frac{i v}{2}\left(\begin{array}{c}
g\left(W_{\mu}^{1}-i W_{\mu}^{2}\right) \\
g W_{\mu}^{3}-g^{\prime} B_{\mu}
\end{array}\right)=\frac{i v}{2}\left(\begin{array}{c}
\sqrt{2} g W_{\mu}^{+} \\
\sqrt{g^{2}+g^{\prime 2}} Z_{\mu}
\end{array}\right),
$$

where we have defined

$$
W_{\mu}^{ \pm} \equiv \frac{W_{\mu}^{1} \mp i W_{\mu}^{2}}{\sqrt{2}}, \quad Z_{\mu} \equiv c_{W} W_{\mu}^{3}-s_{W} B_{\mu}
$$

in terms of the "Weinberg angle" $\theta_{W}$ defined by $\tan \theta_{W}=g^{\prime} / g, 0 \leq \theta_{W} \leq \pi / 2$. The charged and neutral vector bosons $W_{\mu}^{ \pm}$and $Z_{\mu}$ turn then out to have definite mass. We have in fact $\left(D_{\mu}\langle H\rangle\right)^{*}\left(D^{\mu}\langle H\rangle\right)=M_{W}^{2} W_{\mu}^{+} W^{\mu-}+M_{Z}^{2} Z_{\mu} Z^{\mu} / 2$, with the vector boson masses given by

$$
M_{W}^{2}=\frac{g^{2}}{2} v^{2}, \quad M_{Z}^{2}=\frac{g^{2}+g^{\prime 2}}{2} v^{2} \quad \text { and } \quad \frac{G_{F}}{\sqrt{2}}=\frac{g^{2}}{8 M_{W}^{2}}=\frac{1}{4 v^{2}},
$$

determining the electroweak scale in terms of $G_{F}$. The fourth vector boson, $A_{\mu}=$ $s_{W} W_{\mu}^{3}+c_{W} B_{\mu}$, the photon, does not get a mass term, as the corresponding generator is not broken. In order to be able to write the gauge interactions in terms of the vector bosons with definite masses, $W_{\mu}^{ \pm}, Z_{\mu}, A_{\mu}$, it suffices to write the covariant derivative in terms of the latter:

$$
D_{\mu}=\partial_{\mu}+i \frac{g}{\sqrt{2}} W_{\mu}^{+} T^{+}+i \frac{g}{\sqrt{2}} W_{\mu}^{-} T^{-}+i e Q A_{\mu}+i \frac{g}{c_{W}}\left(T_{3}-s_{W}^{2} Q\right) Z_{\mu}
$$

where $e$ is given by $e=g s_{W}=g^{\prime} c_{W}=g g^{\prime} / \sqrt{g^{2}+g^{\prime 2}}$ and we have defined $T^{ \pm}=T_{1} \pm i T_{2}$. From the above expression of the covariant derivative we recover the electromagnetic interactions of the photon, proportional to the electric charge $Q$, and the charged current interactions of the $W^{ \pm}$'s in eq. (9). New, "neutral current" 
interactions involving the $Z$ boson are also predicted proportional to the coupling $\left(g / c_{W}\right)\left(T_{3}-s_{W}^{2} Q\right)$.

We note the relation

$$
\rho \equiv \frac{M_{W}^{2}}{M_{Z}^{2} \cos ^{2} \theta_{W}}=1 \quad \text { (tree level) }
$$

The above relation is not a general result. It depends on the fact that we have broken the electroweak symmetry by means of a $\mathrm{SU}(2)_{L}$ doublet and not, for example, a triplet. Indeed, the experimental verification of the above relation (where the Weinberg angle is independently measured) rules out significant contributions to spontaneous breaking by additional scalar triplets. The relation in eq. (25) receives small perturbative corrections and is related to a $\mathrm{SU}(2)_{L} \times \mathrm{SU}(2)_{R}$ "custodial" symmetry of the Higgs potential.

Let us spell out in greater detail the form of the gauge interactions of the fermions, the vector bosons, and the Higgs. The fermion gauge interactions come from the term

$$
\bar{\Psi} i D_{\mu} \gamma^{\mu} \Psi=\bar{\Psi} i \partial_{\mu} \gamma^{\mu} \Psi-\left(\frac{g}{\sqrt{2}} j_{c}^{\mu} W_{\mu}^{+}+\text {h.c. }\right)-\frac{g}{c_{W}} j_{n}^{\mu} Z_{\mu}-e j_{\mathrm{em}}^{\mu} A_{\mu},
$$

where the charged and neutral currents $j_{c}^{\mu}$ and $j_{n}^{\mu}$ are given by

$$
j_{c}^{\mu}=\overline{\nu_{i L}} \gamma^{\mu} e_{i L}+\overline{u_{i L}} \gamma^{\mu} d_{i L}, \quad j_{n}^{\mu}=\sum_{f} \overline{f_{X}} \gamma^{\mu}\left(T^{3}-s_{W}^{2} Q\right) f_{X} \quad\left(f=\nu_{i}, e_{i}, u_{i}, d_{i}, X=L, R\right) .
$$

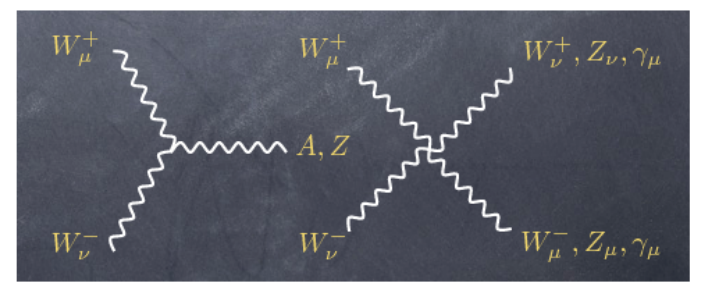

The vector boson gauge self-interactions come from the "Yang-Mills" term:

$$
-W_{\mu \nu}^{a} W^{\mu \nu a} / 4
$$

where

$$
W_{\mu \nu}^{a}=\partial_{\mu} W_{\nu}^{a}-\partial_{\nu} W_{\mu}^{a}-g \epsilon_{a b c} W_{\mu}^{b} W_{\nu}^{c} .
$$

Figure 7: Vector boson gauge self- When expressed in terms of the vector mass interactions. eigenstates $W^{ \pm}, Z, A$, the Yang-Mills term gives trilinear and quartic interactions, as shown in Fig. 7. The existence of the $\gamma W^{+} W^{-}$and $Z W^{+} W^{-}$vertexes has been experimentally established at LEP. Fig. 8 shows the measurement of the cross Section of $e^{+} e^{-} \rightarrow W^{+} W^{-}$and the corresponding theoretical predictions obtained taking into account the exchange of a neutrino only (a), adding the exchange of a photon through the $\gamma W^{+} W^{-}$interactions $(\mathrm{a}+\mathrm{b})$, and further adding the exchange of a $Z$ through the $Z W^{+} W^{-}$vertex $(\mathrm{a}+\mathrm{b}+\mathrm{c})[7]$.

Finally, the Higgs gauge interactions are given in unitary gauge by

$$
\left(\sqrt{2} \frac{h}{v}+\frac{h^{2}}{2 v^{2}}\right)\left(M_{W}^{2} W_{\mu}^{+} W^{\mu-}+\frac{1}{2} M_{Z}^{2} Z_{\mu} Z^{\mu}\right) .
$$




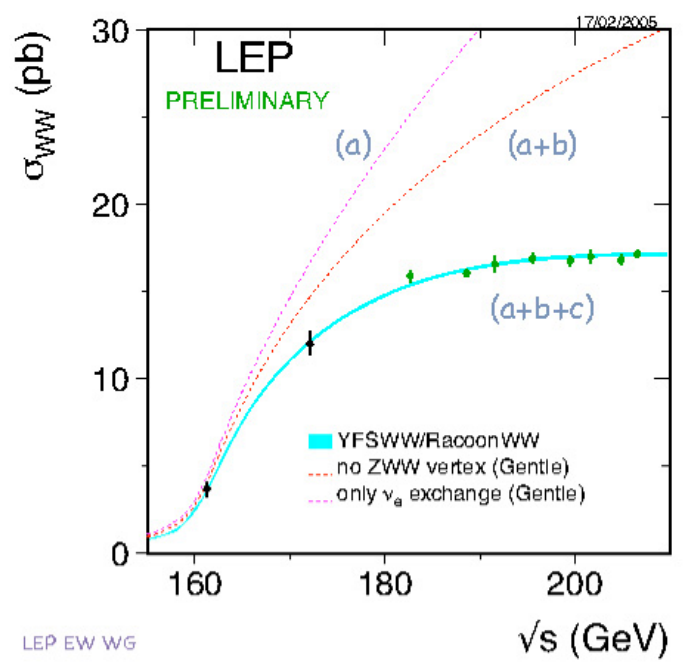

Figure 8: Measurement of the $e^{+} e^{-} \rightarrow$ $W^{+} W^{-}$cross section as a function of the center of mass energy and comparison with different theoretical predictions. dences that, although strictly speaking do not contradict the SM, represent strong hint for physics beyond the SM: the peculiar structure of the SM gauge quantum numbers in Table 1, and neutrino masses. The SM quantum numbers can be nicely understood in terms of grand-unified theories, which also lead to the successful, precise prediction of the strong coupling within supersymmetric models. Neutrino masses can be incorporated in the SM by means of an effective interaction of two lepton doublets and two Higgses. Still, the existence of an effective, non-renormalizable interaction represents a strong hint for new physics arising at a higher scale. There are then a number of theoretical puzzles that do not represent a clear indication for new physics but we would be very happy to understand in terms of physics beyond the SM: the smallness of the electroweak scale compared to the Planck scale, family replication, the existence of small Yukawa couplings and the peculiar pattern of fermion masses and mixings. Finally, the SM has a number of theoretical problems. The naturalness/unitarity problem, related to the stability of the Higgs mass with respect to radiative corrections in the presence of a new high scale (of which we have at least an incontrovertible example: the Planck scale); the similar (from a QFT point of view) problem of the smallness of the cosmological constant. The strong CP-problem.

For all the above reasons we believe that the SM is not the end of the story. I wish the younger generations attending this school to have the opportunity to witness and hopefully play an important role in the many developments to come. 


\section{Acknowledgments}

I would like to thank all the organizers, and Dima in particular, for introducing me to such a special school; the students, the pancake makers, and all the people and fowls involved for the magic atmosphere they contributed to create; and... a hero guy authority in SM.

\section{Bibliography}

[1] C. Amsler et al. [Particle Data Group], Phys. Lett. B 667 (2008) 1.

[2] F. Englert and R. Brout, Phys. Rev. Lett. 13 (1964) 321. P. W. Higgs, Phys. Rev. Lett. 13 (1964) 508. G. S. Guralnik, C. R. Hagen and T. W. B. Kibble, Phys. Rev. Lett. 13 (1964) 585.

[3] S. L. Glashow, Nucl. Phys. 22 (1961) 579. S. Weinberg, Phys. Rev. Lett. 19 (1967) 1264. A. Salam, Originally printed in "Svartholm: Elementary Particle Theory, Proceedings of the Nobel Symposium Held 1968 at Lerum, Sweden", Stockholm $1968,367-377$

[4] E. Fermi, Z. Phys. 88 (1934) 161. G. Gamow and E. Teller, Phys. Rev. 49 (1936) 895. E. C. G. Sudarshan and R. e. Marshak, Phys. Rev. 109 (1958) 1860.

[5] Y. Nambu, Phys. Rev. 117 (1960) 648. J. Goldstone, Nuovo Cim. 19 (1961) 154. J. Goldstone, A. Salam and S. Weinberg, Phys. Rev. 127 (1962) 965.

[6] T. Hambye and K. Riesselmann, Phys. Rev. D 55 (1997) 7255 [arXiv:hep$\mathrm{ph} / 9610272]$.

[7] http://lepewwg.web.cern.ch/ 